\title{
Sexually dimorphic effects of oxytocin receptor gene (OXTR) variants on Harm Avoidance
}

\author{
Trayana Stankova ${ }^{1}$, Peter Eichhammer ${ }^{2}$, Berthold Langguth ${ }^{2}$ and Philipp G Sand ${ }^{2 *}$
}

\begin{abstract}
Background: Recent research has suggested that oxytocin receptor gene (OXTR) variants may account for individual differences in social behavior, the effects of stress and parenting styles. Little is known, however, on a putative role of the gene in heritable temperamental traits.

Methods: We addressed effects of two common OXTR variants, rs237900 and rs237902, on personality dimensions in 99 healthy subjects using the Temperament and Character Inventory.

Results: When sex was controlled for and an OXTR genotype*sex interaction term was included in the regression model, $11 \%$ of the variance in Harm Avoidance could be explained (uncorrected $p \leq 0.01$ ). Female carriers of the minor alleles scored highest, and a novel A217T mutation emerged in the most harm avoidant male participant.

Conclusions: Findings lend support to a modulatory effect of common OXTR variants on Harm Avoidance in healthy caucasian women and invite resequencing of the gene in anxiety phenotypes to identify more explanatory functional variation.
\end{abstract}

Keywords: Oxytocin receptor gene, Personality traits, Sexual dimorphism

\section{Background}

The neuropeptide oxytocin is synthesized in magnocellular neurons of the hypothalamus and is released into the blood stream from the posterior pituitary stimulating smooth muscle contractions during labor and birth. Gprotein-coupled oxytocin receptors (OXTR), however, mediate not only oxytocin peripheral actions in reproduction but also a range of central nervous actions that regulate human social behavior [1]. From investigations of the gene encoding OXTR on human chromosome 3p26, associations have emerged with disrupted affialitive behavior [2], the seeking of emotional support [3], and attachment anxiety in women [4], among other traits. At the brain structural level, variation in the OXTR gene would appear to affect amygdala volume [5], indicating a possible vulnerability to the adverse effects of stress [6]. Functional imaging experiments strongly suggest that stress-induced dopamine release and increased attachment anxiety are both under control of OXTR variation [7]. In animals, patterns of OXTR

\footnotetext{
* Correspondence: philipp.sand@klinik.uni-regensburg.de

${ }^{2}$ Department of Psychiatry and Psychotherapy, University of Regensburg,

Universitaetsstrasse 84, 93053 Regensburg, Germany

Full list of author information is available at the end of the article
}

expression in the central nervous system have long suggested a contribution of OXTR to the regulation of anxiety [8] and to defensive maternal behavior [9].

Although the spectrum of OXTR-related candidate phenotypes has thus greatly expanded, it is still unclear which personality traits should be considered a part of this spectrum. Animal models have implicated oxytocin effects in two temperaments, Novelty Seeking (NS) and Harm Avoidance (HA) [10], but a study of human OXTR variants has pointed to a third temperamental factor, Reward Dependence (RD) [11]. Most recently, a role has been proposed for OXTR variation in predicting optimism and self-esteem [12,13], i.e. traits negatively correlated with HA [14]. According to Cloninger's psychobiological model of temperament and character, NS is related to behavioral activation, $\mathrm{HA}$ to behavioral inhibition or anxiety-proneness, and RD to behavioral maintenance. In order to shed more light on this issue, we readdressed the impact of OXTR variation on personality dimensions using the Temperament and Character Inventory (TCI) [15].

\section{Materials and methods}

106 healthy unrelated Caucasian subjects were recruited among hospital staff and their friends in the Regensburg 
area. All provided informed consent, donated a blood sample, and were administered the 226-item German Temperament and Character Inventory (TCI) version 8 [15]. The TCI evaluates four higher order temperament and three higher order character traits. Each of the seven traits is multifaceted, i.e. it consists of lower order components. On the whole, the TCI is made up of twentyfive facets (12 facets of temperament and 13 facets of character) of which we examined only the genetically independent main temperamental factors, Novelty Seeking, Harm Avoidance, Reward Dependence, and Persistence [16]. After excluding incomplete questionnaires, 99 volunteers (48 men and 51 women, mean age $29.1 \pm 7.4$ years and $28.7 \pm 7.7$ years, respectively) were genotyped for two OXTR variants, rs237900 and rs237902 by Sanger sequencing (Primers: 5'-GCAGGTGCA CATCTTCTCTC-3; forward, and 5'-GGAGTCCCTT GAACCTGTTT-3, reverse). The variants were selected for having previously been implicated in Novelty Seeking (rs237902, see [17]), for a heterozygosity of $>0.4$ (both variants), and for their inclusion in major commercial SNP arrays (rs237900). Sequence alignments were conducted with DNA Dynamo 1.0 (Blue Tractor Software, UK). STATA 8.0 (Stata Corporation, College Station, TX, USA) was used for descriptive statistics and multiple regression. The level of statistical significance was set at $\mathrm{p}<0.05$. Evolutionary conservation in 46 mammals was assessed with a phylogenetic hidden Markov model-based method, phastCons [18]. A probabilistic classifier incorporating secondary structure information (PolyPhen2) was used to refine this estimate for nonsynonymous OXTR variants.

\section{Results}

TCI scores conformed to a normal distribution for NS, HA, and RD, but not for PS. Consistent with previous research [19], PS scores were therefore log-transformed before statistical analyses to ensure Gaussian distributions across all scales (Shapiro-Wilk test of transformed data $p>0.67$; reported means and SDs are not transformed for ease of interpretation). Effects of rs237900 and rs237902 on the TCI temperamental scales were non-significant when data from men and women were pooled $(F<1.25, p>0.26)$. However, when sex was controlled for, both OXTR variants under study predicted Harm Avoidance in multiple regression models $(p=0.01$ for $\mathrm{rs} 237902, p=0.006$ for rs237900). Considering moderate to strong intermarker linkage disequilibrium $\left(r^{2}=0.75\right)$, we limited our analyses to rs237900 (Table 1). To avoid trade-offs in power, CT and TT genotypes were collapsed into $\mathrm{T}$ carriers and were then contrasted with the remaining subjects under the assumption of a recessive mode of inheritance (mean $\mathrm{HA}$ scores $\pm \mathrm{SD}$ : $\mathrm{CC}=13.4 \quad \pm 4.7, \quad \mathrm{CT}=14.3 \quad \pm 6.3, \quad \mathrm{TT}=14.3 \quad \pm 5.1)$.
Table $1 \mathrm{TCl}$ temperament scores (mean \pm SD) for 99 men and women, stratified by OXTR genotype

\begin{tabular}{lcccc}
\hline OXTR genotype $^{\mathbf{a}}$ & NS & HA & RD & PS \\
\hline male T-allele carriers $(N=27)$ & $20.0 \pm 6.1$ & $12.7 \pm 5.4$ & $15.0 \pm 3.7$ & $4.4 \pm 2.1$ \\
female T-allele carriers $(N=29)$ & $22.3 \pm 6.1$ & $16.4 \pm 5.4$ & $16.5 \pm 2.9$ & $3.4 \pm 1.9$ \\
male non-carriers $(N=20)$ & $21.5 \pm 6.1$ & $11.9 \pm 4.5$ & $16.5 \pm 3.6$ & $4.1 \pm 1.8$ \\
female non-carriers $(N=21)$ & $22.8 \pm 4.4$ & $14.5 \pm 5.0$ & $15.4 \pm 3.2$ & $4.0 \pm 1.9$ \\
effects of predictor variables & & & & \\
$F(2,94)$ [sex rs237900] & 1.615 & 5.460 & 0.215 & 1.396 \\
$P_{\text {[sex rs237900] }}$ & 0.204 & 0.005 & 0.807 & 0.253 \\
$P_{\text {[corrected] }}$ & 1.000 & 0.046 & 1.000 & 1.000 \\
$F(3,93)$ [including sex*rs237900] & 1.144 & 3.693 & 1.407 & 1.327 \\
$P_{\text {[including sex*rs237900] }}$ & 0.335 & 0.014 & 0.246 & 0.270 \\
\hline
\end{tabular}

When sex is controlled for and a recessive mode of inheritance is assumed, rs237900 predicts Harm Avoidance in additive and multiplicative regression models.

significance tests refer to log-transformed $\mathrm{TCl}$ scores.

${ }^{a}$ results are shown for rs237900, 2 unreadable $A B I$ trace files. $\mathrm{NS}=$ Novelty Seeking, $\mathrm{HA}=\mathrm{Harm}$ Avoidance, $\mathrm{RD}=$ Reward Dependence, $\mathrm{PS}=$ Persistence.

Following a Bonferroni correction for eight separate regressions (effects of genotype on four temperamental scales with and without sex as a second factor), significance was still achieved $(p=0.045)$. Female carriers of the minor allele $(\mathrm{T})$ scored higher on Harm Avoidance than did all other subjects. Even though only sex appeared to exert a main effect in the additive model (Figure 1), the variance explained by both regressor variables reached $11 \%$ when an interaction term was included (Table 1, 10\% assuming no interaction). No noteworthy effect was observed on Novelty Seeking, Reward Dependence or Persistence.

Detailed examination of the sequence chromatograms revealed one carrier of a novel synonymous variant, Y200Y (TAC > TAT), plus one carrier of a novel

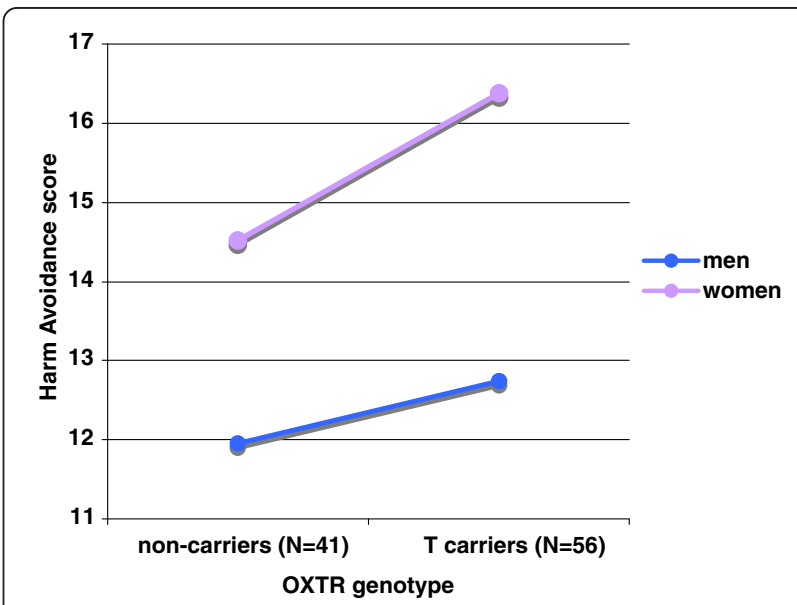

Figure 1 Interaction of OXTR genotype ( $t=1.26, p=0.20)$ and sex $(t=-3.05, p=0.003)$ as predictors of Harm Avoidance mean scores. 
missense mutation, A217T (GCT $>$ ACT). The latter coincided with the highest Harm Avoidance score of all male subjects, and the 98th percentile of pooled Harm Avoidance scores under a Gaussian distribution. To obtain more detailed information on the prevalence of this substitution in the general population, we screened an additional set of previously recruited control subjects (healthy by self-report, no TCI data available) originating from the greater Regensburg area using the same experimental protocol ( $N=396,172$ men and 224 women). They comprised university students, other hospital employees, and their acquaintances. No further carriers were identified, lowering the total allele frequency to 0.001. A217T maps to a region encoding the fifth OXTR transmembrane domain (TMD), delimited by residues 203 and 225. Evolutionary conservation of this region in mammals is high (Figure 2) and in silico predictions indicated that the variant is possibly damaging (PolyPhen2 score 0.46). Two known OXTR variants, rs4686302, rs61740241, were confirmed in the course of screening the extension sample, plus two novel intronic variants $(\mathrm{T}>\mathrm{C}$, chr3: 8,808,677 and $\mathrm{G}>\mathrm{A}$, chr3: 8,808,925). Two subjects also carried one novel OXTR missense variant each, E242K (GAG > AAG) and
G252A (GGG > GCG) that, however, do not map to any TMD and appear benign (PolyPhen2 score $<0.01$ ).

\section{Discussion}

The present results extend previous work on OXTR sequence variants' role in predicting facets of human behavior. Under the assumption of sexually dimorphic effects, an association was observed for two substitutions and Harm Avoidance, with female carriers of the minor alleles scoring highest.

The possible implications of this observation are three-fold:

From an evolutionary perspective, oxytocin control of Harm Avoidance in humans is relevant to parental care and related behaviors. According to Cloninger, harm avoidant reaction patterns have arisen from multifactorial phylogenetic mechanisms which in their 'optima' are advantageous to the adaptation and survival of the individual [20]. Human offspring require more intense care and protection from hazards in early life than other species. Both the fine-tuning of appraisal mechanisms that detect potential threats to the offspring and the initiation of alarm responses in parents are key to averting hazards. There is a growing concern, however, that high

(a)

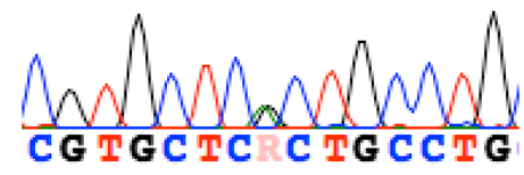

(b)

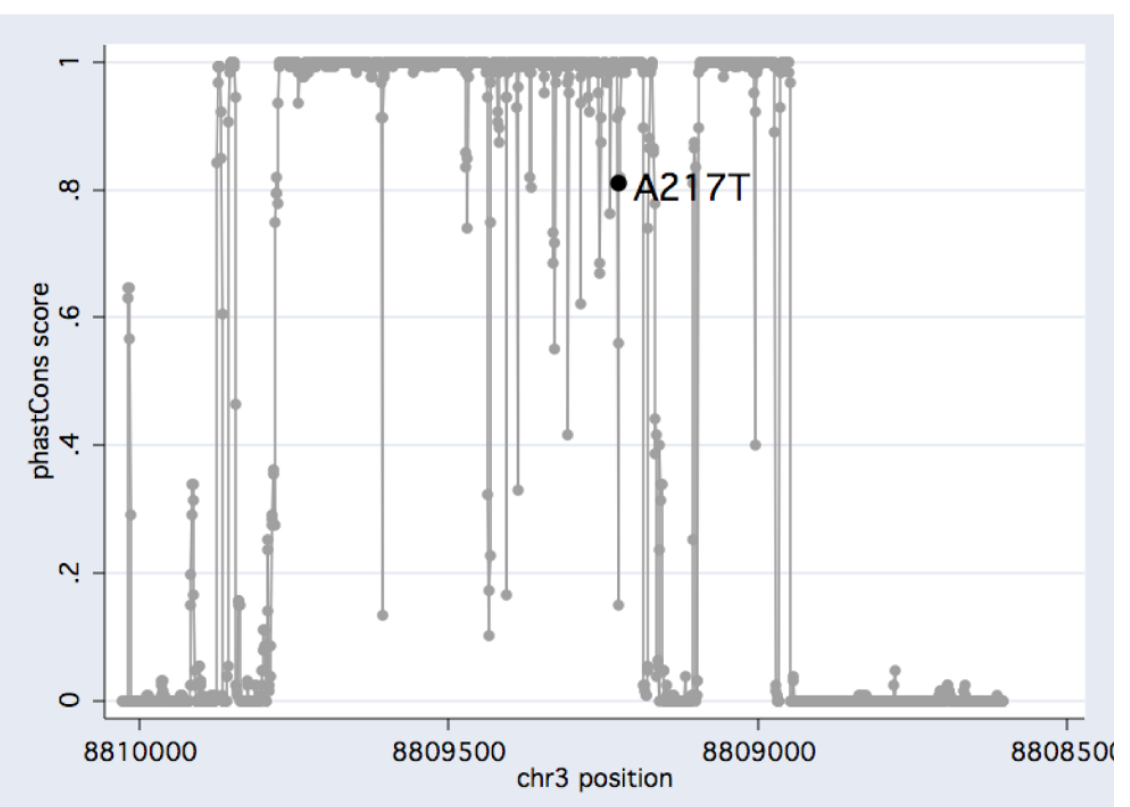

Figure 2 Chromatogram (a) and evolutionary conservation plot (b) of a newly identified OXTR mutation, A217T, in a subject scoring on the 98th percentile for Harm Avoidance. Basewise conservation of the amplicon sequence is plotted from $5^{\prime}$ to $3^{\prime}$ against the physical position on chromosome 3 using 46 placental mammals featured in the UCSC Genome Browser. A217T maps to a highly conserved region encoding the 5th OXTR transmembrane domain. 
levels of anxiety or excessive harm avoidant behavior in parents may be counter-productive and, in some agegroups, may even augment childrens' levels of anxiety [21]. Oxytocin exerts anxiolytic effects that can balance and optimize maternal defense. Consistent with this view, breast-feeding women show lower systolic blood pressure reactivity to aggressive encounters than do formula-feeding women [22]. Similar observations have been made during lactation using cold pressor and mental arithmetic stressor tasks [23]. Provided that OXTR are involved in these functionalities, and provided the present association is genuine, women carrying the minor OXTR alleles could be less susceptible to the anxiety-attenuating effects of oxytocin than subjects carrying only wild-type alleles and, possibly, more prone to responding aggressively to detected threats. While a certain measure of heightened reactivity to stressful stimuli should benefit the defense of offspring, excessive emotional lability is liable to interfere with sensitive parenting [24]. Sex-specific responses to oxytocin could be one reason why HA is gender-sensitive [25]. Until this is confirmed, the absence of a main effect of genotype on HA scores in men cautions against overrating the predictive role of OXTR in shaping personality traits.

From a more general point of view, the gene encoding OXTR has been implicated in the responsiveness to social support as a buffer against stress [26]. This extends the reciprocal interaction between serotonin and social behavior to the oxytocinergic system [24,27]. To specifically evaluate gene $\mathrm{x}$ environment interactions of the SNPs examined, however, more work is needed in a context of psychosocial stress exposure. Theoretically, a putative loss of OXTR function could be mediated either by the $\mathrm{T}$ alleles, or by mutations that are in linkage disequilibrium with these alleles. Of the two variants addressed here, rs 237900 is intronic, and its functionality is uncertain. Rs237902 stands for a synonymous substitution at residue 230 and has the potential to interfere with gene expression, e.g. by disrupting motifs recognized by transcription factors, or by affecting posttranscriptional regulatory mechanisms. Confirmatory investigations in larger samples are still required, together with an elucidation of the mechanism by which the endocrine response is altered.

In contrast to the results described earlier for rs53576 [11], we failed to identify significant genetic effects on the personality dimension of Reward Dependence. This could be due to a difference in sample size, or to using different markers. Others have shown that rs237902 and rs53576 map to two different haplotype blocks in the Caucasian population [2]. Balancing of Harm Avoidance and Reward Dependence would appear to facilitate response selection mechanisms in parental care. On the one hand, sources of reward trigger heightened distress when the attachment object is being threatened. Thus reward-related reactivity of mothers to images of infants (as measured by activation of the ventral striatum) varies with cues of potential harm $[28,29]$. On the other hand, reward responses are believed to buffer otherwise deleterious levels of heightened anxiety and stress [30]. On these grounds, common OXTR variants could serve to predict Harm Avoidance and Reward Dependence, depending on the markers used, and on the assumptions made in the respective models. It is noteworthy that sexually dimorphic effects of OXTR variants are emerging in the sensitivity to pain [7], a composite trait integrating, in turn, measures of Harm Avoidance and Reward Dependence [31].

Finally, identification of a novel A217T mutation in a highly harm avoidant subject may be a chance finding. Whether a putative loss of receptor function is aggravated any further by this rare substitution is unresolved. Its location in a highly conserved region argues against a neutral effect on the OXTR protein and should therefore justify inclusion in future investigations of target phenotypes.

\section{Summary and conclusion}

The experiments discussed here are the first to rationalize a role of the oxytocin receptor gene in Harm Avoidance and incentivize resequencing the OXTR gene in conditions marked by an increased sensitivity to perceived threat. We acknowledge that extrapolations of our findings to oxytocin-mediated adaptive responses in the context of parenting, or to stress responses in general, are, at this stage, largely tentative. OXTR variants may predispose to developing less specific behavioral symptoms by interfering with visual attention and awareness [32]. Moreover, most investigations of parenting have focused on maternal behavior. More detailed assessments are now warranted to replicate the association of OXTR with HA, adding to the emerging association of OXTR variants with parenting styles in mothers and fathers [33].

\section{Competing interests}

The authors declare that they have no competing interests.

\section{Authors' contributions}

PGS designed the study and wrote the protocol. TS conducted the experiments and assisted PGS with statistical analysis. PE supervised the recruitment of subjects who took part in the study. BL and TS managed the literature searches, analyzed the data and helped to draft the manuscript. All authors contributed to and have approved the final manuscript.

\section{Author details}

${ }^{1}$ Experimental and Clinical Neurosciences Graduate Program, University of Regensburg, Regensburg, Germany. ${ }^{2}$ Department of Psychiatry and Psychotherapy, University of Regensburg, Universitaetsstrasse 84, 93053 Regensburg, Germany. 
Received: 20 February 2012 Accepted: 11 July 2012

Published: 30 July 2012

\section{References}

1. Heinrichs M, von Dawans B, Domes G: Oxytocin, vasopressin, and human social behavior. Front. Neuroendocrinol 2009, 30:548-557. doi:10.1016/j. yfrne.2009.05.005.

2. Wermter AK, Kamp-Becker I, Hesse P, Schulte-Körne G, Strauch K, Remschmidt $\mathrm{H}$ : Evidence for the involvement of genetic variation in the oxytocin receptor gene (OXTR) in the etiology of autistic disorders on high-functioning level. Am J Med Genet B Neuropsychiatr Genet 2010, 153B:629-639. doi:10.1002/ajmg.b.31032.

3. Kim HS, Sherman DK, Sasaki JY, Xu J, Chu TQ, Ryu C, Suh EM, Graham K Taylor SE: Culture, distress, and oxytocin receptor polymorphism (OXTR) interact to influence emotional support seeking. Proc Natl Acad Sci U S A 2010, 107:15717-15721. doi:10.1073/pnas.1010830107.

4. Chen FS, Johnson SC: An oxytocin receptor gene variant predicts attachment anxiety in females and autism-spectrum traits in males. Soc Psychol. Person. Sci. 2012, 3:93-99. doi:10.1177/1948550611410325.

5. Inoue H, Yamasue H, Tochigi M, Abe O, Liu X, Kawamura Y, Takei K, Suga M, Yamada H, Rogers MA, Aoki S, Sasaki T, Kasai K: Association between the oxytocin receptor gene and amygdalar volume in healthy adults. Biol Psychiatry 2010, 68:1066-1072. doi:10.1016/j.biopsych.2010.07.019.

6. Furman DJ, Chen MC, Gotlib IH: Variant in oxytocin receptor gene is associated with amygdala volume. Psychoneuroendocrinology 2011, 36:891-897. doi:10.1016/j.psyneuen.2010.12.004.

7. Love TM, Enoch MA, Hodgkinson CA, Peciña M, Mickey B, Koeppe RA, Stohler CS, Goldman D, Zubieta JK: Oxytocin gene polymorphisms influence human dopaminergic function in a sex-dependent manner. Biol Psychiatry 2012, 72:198-206. doi:10.1016/j.biopsych.2012.01.033.

8. Bale TL, Davis AM, Auger AP, Dorsa DM, McCarthy MM: CNS region-specific oxytocin receptor expression: importance in regulation of anxiety and sex behavior. J Neurosci 2001, 21:2546-2552.

9. Bosch OJ, Meddle SL, Beiderbeck DI, Douglas AJ, Neumann ID: Brain oxytocin correlates with maternal aggression: link to anxiety. J Neurosci 2005, 25:6807-6815.

10. Ray J, Hansen S: Temperament in the rat: sex differences and hormonal influences on harm avoidance and novelty seeking. Behav Neurosci 2004, 118:488-497. doi:10.1037/0735-7044.118.3.488.

11. Tost H, Kolachana B, Hakimi S, Lemaitre H, Verchinski BA, Mattay VS, Weinberger DR, Meyer-Lindenberg A: A common allele in the oxytocin receptor gene (OXTR) impacts prosocial temperament and human hypothalamic-limbic structure and function. Proc Natl Acad Sci U S A 2010, 107:13936-13941. doi:10.1073/pnas.1003296107.

12. Saphire-Bernstein S, Way BM, Kim HS, Sherman DK, Taylor SE: Oxytocin receptor gene (OXTR) is related to psychological resources. Proc Natl Acad Sci U S A 2011, 108:15118-15122. doi:10.1073/pnas.1113137108.

13. Cornelis MC, Glymour MM, Chang SC, Tchetgen EJ, Liang L, Koenen KC, Kang JH, Pasquale LR, Rimm EB, Kawachi I, Kubzansky LD: Oxytocin receptor (OXTR) is not associated with optimism in the Nurses' Health Study. Mol Psychiatry 2012, doi:10.1038/mp.2011.178.

14. Krebs $H$, Weyers $P$, Janke $W$ : Validation of the German version of Cloninger's TPQ: Replication and correlations with stress coping, mood measures and drug use. Pers. Indiv. Differ 1998, 24:805-814. doi:10.1016/ S0191-8869(98)00008-7.

15. Berth $\mathrm{H}$, Cloninger CR, Przybeck TR, Svrakic DM, Wetzel RD: Das Temperament- und Charakter-Inventar (TCl). Diagnostica 2001, 47:51-53.

16. Melke J, Westberg L, Nilsson S, Landen M, Soderstrom H, Baghaei F, Rosmond R, Holm G, Björntorp P, Nilsson LG, Adolfsson R, Eriksson E: A polymorphism in the serotonin receptor $3 \mathrm{~A}$ (HTR3A) gene and its association with harm avoidance in women. Arch Gen Psychiatry 2003, 60:1017-1023

17. Comings DE, Gade-Andavolu R, Gonzalez N, Wu S, Muhleman D, Blake H, Mann MB, Dietz G, Saucier G, MacMurray JP: A multivariate analysis of 59 candidate genes in personality traits: the temperament and character inventory. Clin Genet 2000, 58:375-385. doi:10.1034/j.1399-0004.2000.580508.X

18. Rhead B, Karolchik D, Kuhn RM, Hinrichs AS, Zweig AS, Fujita PA, Diekhans M, Smith KE, Rosenbloom KR, Raney BJ, Pohl A, Pheasant M, Meyer LR, Learned K, Hsu F, Hillman-Jackson J, Harte RA, Giardine B, Dreszer TR, Clawson H, Barber GP, Haussler D, Kent WJ: The UCSC Genome Browser database: update 2010. Nucleic Acids Res 2010, 38:D613-D619. doi:10.1093/nar/gkp939.
19. Fassino S, Amianto F, Gastaldi F, Abbate-Daga G, Brambilla F, Leombruni P: Personality trait interactions in parents of patients with borderline personality disorder: a controlled study using the Temperament and Character Inventory. Psychiatry Res 2009, 165:128-136. doi:10.1016/j. psychres.2007.10.010

20. Cloninger CR: A unified biosocial theory of personality and its role in the development of anxiety states. Psychiatr Dev 1986, 4:167-226.

21. van der Bruggen CO, Stams GJ, Bögels SM: Research review: the relation between child and parent anxiety and parental control: a meta-analytic review. J Child Psychol Psychiatry 2008, 49:1257-1269. doi:10.1111/j.14697610.2008.01898.x.

22. Hahn-Holbrook J, Holt-Lunstad J, Holbrook C, Coyne SM, Lawson ET: Maternal defense: breast feeding increases aggression by reducing stress. Psychol Sci 2011, 22:1288-1295. doi:10.1177/0956797611420729.

23. Mezzacappa ES, Kelsey RM, Katkin ES: Breast feeding, bottle feeding, and maternal autonomic responses to stress. J Psychosom Res 2005, 58:351-365. doi:10.1016/j.jpsychores.2004.11.004.

24. Bakermans-Kranenburg MJ, van ljzendoorn MH: Oxytocin receptor (OXTR) and serotonin transporter (5-HTT) genes associated with observed parenting. Soc Cogn Affect Neurosci 2008, 3:128-134. doi:10.1093/scan/ nsn004.

25. Li Y, Qin W, Jiang T, Zhang Y, Yu C: Sex-Dependent Correlations between the Personality Dimension of Harm Avoidance and the Resting-State Functional Connectivity of Amygdala Subregions. PLoS One 2012, 7:e35925. doi:10.1371/journal.pone.0035925.

26. Chen FS, Kumsta R, von Dawans B, Monakhov M, Ebstein RP, Heinrichs M: Common oxytocin receptor gene (OXTR) polymorphism and social support interact to reduce stress in humans. 2011, 108:19937-19942. doi:10.1073/pnas.1113079108.

27. Marazziti D, Baroni S, Giannaccini G, Betti L, Massimetti G, Carmassi C, Catena-Dell'osso M: A link between oxytocin and serotonin in humans: Supporting evidence from peripheral markers. Eur. Neuropsychopharmacol. 2012, doi:10.1016/j.euroneuro.2011.12.010.

28. Strathearn $L$ : Maternal neglect: oxytocin, dopamine and the neurobiology of attachment. J. Neuroendocrinol. 2011, 23:1054-1065. doi:doi: 10.1111/ j.1365-2826.2011.02228.x.

29. Strathearn L, Fonagy P, Amico J, Montague PR: Adult attachment predicts maternal brain and oxytocin response to infant cues. Neuropsychopharmacology 2009, 34:2655-2666. doi:10.1038/npp.2009.103.

30. Ulrich-Lai YM, Christiansen AM, Ostrander MM, Jones AA, Jones KR, Choi DC, Krause EG, Evanson NK, Furay AR, Davis JF, Solomon MB, de Kloet AD, Tamashiro KL, Sakai RR, Seeley RJ, Woods SC, Herman JP: Pleasurable behaviors reduce stress via brain reward pathways. Proc. Natl. Acad. Sci USA 2010, 107:20529-20534. doi:10.1073/pnas.1007740107.

31. Pud D, Eisenberg E, Sprecher E, Rogowski Z, Yarnitsky D: The tridimensional personality theory and pain: harm avoidance and reward dependence traits correlate with pain perception in healthy volunteers. Eur. J. Pain 2004, 8:31-38. doi:10.1016/S1090-3801(03)00065-X.

32. Guastella AJ, Carson DS, Dadds MR, Mitchell PB, Cox RE: Does oxytocin influence the early detection of angry and happy faces. Psychoneuroendocrinology 2009, 34:220-225. doi:10.1016/j. psyneuen.2008.09.001.

33. Feldman R, Zagoory-Sharon O, Weisman O, Schneiderman I, Gordon I, Maoz R, Shalev I, Ebstein RP: Sensitive Parenting Is Associated with Plasma Oxytocin and Polymorphisms in the OXTR and CD38 Genes. Biol Psychiatry 2012, 72:175-181. doi:10.1016/j.biopsych.2011.12.025.

doi:10.1186/2042-6410-3-17

Cite this article as: Stankova et al: Sexually dimorphic effects of oxytocin receptor gene (OXTR) variants on Harm Avoidance. Biology of Sex Differences 2012 3:17. 